\title{
In vitro activity of a G-quadruplex- stabilizing small molecule that synergizes with Navitoclax to induce cytotoxicity in acute myeloid leukemia cells
}

Justin J. Montoya ${ }^{1,2^{*}}$ (D) Megan A. Turnidge $e^{1,2,3,4}$, Daniel H. Wai ${ }^{1,2}$, Apurvi R. Patel ${ }^{1,2,5}$, David W. Lee ${ }^{1,2}$, Vijay Gokhale ${ }^{6}$, Laurence H. Hurley ${ }^{6}$, Robert J. Arceci, ${ }^{1,25^{\wedge}}$, Cynthia Wetmore ${ }^{1,2,5}$ and David O. Azorsa ${ }^{1,2,7}$

\begin{abstract}
Background: Acute Myeloid Leukemia (AML) is a malignancy of myeloid precursor cells that arise from genomic alterations in the expression of key growth regulatory genes causing cells to assume an undifferentiated state and continue to proliferate. Recent efforts have focused on developing therapies that target specific protein products of aberrantly expressed genes. However, many of the identified proteins are difficult to target and thought to be "undrugable" because of structural challenges, protein overexpression, or mutations that confer resistance to therapy. A novel technology that circumvents some of these issues is the use of small molecules that stabilize secondary DNA structures present in the promoters of many potential oncogenes and modulate their transcription.

Methods: This study characterizes the in vitro activity of the G-quadruplex-stabilizing small molecule GQC-05 in AML cells. The effect of GQC-05 on three AML cell lines was analyzed using viability and apoptosis assays. GQC-05 has been shown to down-regulate MYC through G-quadruplex stabilization in Burkitt's lymphoma cell lines. MYC expression was evaluated through $\mathrm{GPCR}$ and immunoblotting in the three AML cell lines following the treatment of GQC-05. In order to identify other therapeutic agents that potentiate the activity of GQC-05, combination drug screening was performed. The drug combinations were validated using in vitro cytotoxicity assays and compared to other commonly used chemotherapeutic agents.
\end{abstract}

Results: GQC-05 treatment of KG-1a, CMK and TF-1 cells decreased cell viability and resulted in increased DNA damage and apoptosis. Additionally, treatment of KG-1a, CMK and TF-1 with GQC-05 resulted in decreased expression of MYC mRNA and protein, with a more pronounced effect in KG-1a cells. Combination drug screening identified the Bcl-2/Bcl$X_{L}$ inhibitor Navitoclax as a compound that potentiated GQC-05 activity. Co-treatment with GQC-05 and Navitoclax showed a synergistic decrease in cell viability of AML cells as determined by Chou-Talalay analysis, and induced more DNA damage, apoptosis, and rapid cytotoxicity. The cytotoxicity induced by GQC-05 and Navitoclax was more potent than that of Navitoclax combined with either cytarabine or doxorubicin.

Conclusion: These results suggest that the G-quadruplex stabilizing small molecule GQC-05 induces down regulated MYC expression and DNA damage in AML cells. Treatment with both GQC-05 with a BCl-2/BCl-X inhibitor Navitoclax results in increased cytotoxic activity, which is more pronounced than Navitoclax or GQC-05 alone, and more significant than Navitoclax in combination with cytarabine and doxorubicin that are currently being used clinically.

Keywords: MYC, G-quadruplex, AML, BCl-2, BCl-X, Navitoclax

\footnotetext{
* Correspondence: justinjmontoya@email.arizona.edu

'The Institute of Molecular Medicine at Phoenix Children's Hospital, $475 \mathrm{~N}$

5th Street, Phoenix, AZ 85004, USA

${ }^{2}$ Department of Child Health, The University of Arizona College of

Medicine-Phoenix, 475 N 5th Street, Phoenix, AZ 85004, USA

Full list of author information is available at the end of the article
}

(c) The Author(s). 2019 Open Access This article is distributed under the terms of the Creative Commons Attribution 4.0 International License (http://creativecommons.org/licenses/by/4.0/), which permits unrestricted use, distribution, and reproduction in any medium, provided you give appropriate credit to the original author(s) and the source, provide a link to the Creative Commons license, and indicate if changes were made. The Creative Commons Public Domain Dedication waiver (http://creativecommons.org/publicdomain/zero/1.0/) applies to the data made available in this article, unless otherwise stated. 


\section{Background}

Acute Myeloid Leukemia (AML) is a malignancy of poorly differentiated myeloid precursor cells that proliferate robustly, leading to abnormal hematopoiesis and eventually to bone marrow failure [1]. Genomic analysis has demonstrated that AML is composed of a heterogeneous population of malignant cells with numerous genetic complexities [2, 3]. These genetic aberrations often lead to dysregulation of proto-oncogenes such as CREB, MYC, EVi-1, MN-1 [4,5], resulting in difficult to treat tumor cells and poor clinical outcome [6].

G-quadruplexes (G4) are secondary four-stranded helical structures of DNA comprised of guanine-rich nucleic acids that are enriched in the promoter regions of many genes. G4 are involved in many cellular processes including DNA replication, gene expression, telomere maintenance and apoptosis [7, 8]. Many genomic sequences contain areas of guanine rich regions that have the ability to self-associate to form G4, which are more thermodynamically stable than double stranded DNA [8]. It has been estimated that nearly $50 \%$ of human genes contain G4s near their promotor regions, and are more frequently found near the promoters of oncogenes or regulatory genes than near housekeeping genes [9]. Since a dynamic equilibrium occurs between the promoter's transcriptionally active single stranded, double stranded, and G-quadruplex forms, there exists the opportunity to repress gene transcription by stabilizing the G-quadruplex structure. Stabilization of G-quadruplex structures have been described previously in vitro using the porphyrin molecule TMPyP4 [10] and the ellipticine analog GQC-05 [11]. One of the first reports that G4s near promotor regions can effect gene expression arose from studies of the c-MYC oncogene, demonstrating that mutation of the endogenous G4 sequence or addition of a G4-stabilizing ligand altered transcription in vivo [10].

MYC regulates many hallmarks of cancer such as cell growth, proliferation, cell-cycle, apoptosis, and cell differentiation, making it a potent oncogene when dysregulated [12] and an attractive, but as yet, "undruggable" therapeutic target [13]. The compound GQC-05 was shown to stabilize the G-quadruplex in the MYC promoter - among other growth regulatory genes - and also repress its transcription in Burkitt's lymphoma [11].

In this present study, we sought to determine the effects GQC-05 on the expression of MYC and other genes, and to characterize the cellular consequences of AML cells exposed to GQC-05. We found a varied cytotoxic activity of GQC-05 in AML cells and we sought to characterize the mechanism of cell death induced by GQC-05. Furthermore, we completed a drug screen to identify potentiators of GQC-05 activity and demonstrated a novel synergy when GQC-05 was combined with the $\mathrm{Bcl}-2 / \mathrm{Bcl}-\mathrm{X}_{\mathrm{L}}$ inhibitor Navitoclax. These studies also demonstrate that GQC-05 can inhibit MYC expression as previously seen in Burkitt's lymphoma [12]. GQC-05 also induces DNA damage response and induced cytotoxic activity that was increased by the addition of Navitoclax, thereby increasing its potential as therapeutic anti-cancer agent.

\section{Methods \\ Cell culture}

All cell lines were authenticated using Short Tandem Repeat (STR) analysis by the University of Arizona genomics core. The CMY [14], CMK [15], and CMS [16] cell lines were a generous gift from Dr. Jeffrey W. Taub, Wayne State University. The KG-1a cell line was grown in IMDM media (Corning) supplemented with 20\% Fetal Bovine Serum (FBS; Atlas Biologicals), 1\% L-Glutamine (Caisson Labs), and 1\% penicillin/streptomycin (Gibco). The UT7epo cells were grown in similar IMDM media that was supplemented with $1 \mathrm{U} / \mathrm{mL}$ recombinant erythropoietin (rhEPO; R\&D Systems). The Molm-13, Kasumi-1, CMY, NB4, TF-1, M-07e, CMK, HEL, THP-1, U937, AML-193, and CMS cells were grown in RPMI 1640 (Corning) with 10\% FBS and 1\% penicillin/streptomycin and L-glutamine. The RPMI growth media for TF- 1 and M-07e was supplemented with $10 \mathrm{ng} / \mathrm{mL}$ granulocyte macrophage colonystimulating factor (GMCSF; R\&D systems), and the media for AML-193 contained $2 \mathrm{ng} / \mathrm{mL}$ GMCSF as well as $5 \mu \mathrm{g} /$ mL Insulin Transferrin Selenium A (ITS; Gibco). PBMCs were isolated from whole blood by density centrifugation using Ficoll (GE Life Sciences) and grown in RPMI (10\% FBS) supplemented with $10 \mathrm{ng} / \mathrm{mL}$ IL-2 (R\&D Systems). All cells were grown at $37^{\circ} \mathrm{C}$ with $5 \% \mathrm{CO}_{2}$. For 6 well plate assays, cells were plated at 1,500,000 cells/well (KG1a and TF-1) or 1,000,000 cells/well (CMK). Cells were allowed to grow overnight before treatment.

\section{Antibodies, primers, and compounds}

Primary antibodies for MYC (Rabbit mAb \#5605), Bcl-2 (Rabbit mAb \#2870, Mouse mAb \#15071), phosphohistone H2A.X (Ser139) (Rabbit Ab \#2577) and PARP (Rabbit mAb \#9532) were purchased from Cell Signaling Technology. The GAPDH (Mouse mAb sc-166,545) primary antibody was purchased from Santa Cruz Biotechnology. Secondary Rabbit and Mouse antibodies used for chemi-luminescence were obtained from Jackson Immunoresearch. Secondary antibodies IRDye $800 \mathrm{CW}$ GAR and IRDye ${ }^{\circ}$ 680RD GAM used for near infrared western blot detection were obtained from LI-COR Biosciences.

Gene specific qPCR primers for MYC (Forward: $5^{\prime}$ GCCCACCACCAGCAGCGACTC-3', Reverse: 5' GCACCTCTTGAGGACCAGTGG-3'), YWHAZ (Fwd: 5'-AGAGAAAGCCTGCTCTCTTGC-3', Rvs: 5' AGGAGTGGGTGTCGCTGTTG-3') were obtained from Life Technologies. 
The G-quadruplex stabilizing compound GQC-05 was synthesized as previously described [11] at the School of Pharmacy, University of Arizona. The small molecules Navitoclax, (+)-JQ1, doxorubicin and cytarabine were obtained from Selleckchem (Houston, TX).

\section{Drug dose response viability assays}

To determine the cytotoxic effects of compounds, AML cells (2000 cells/well) were plated into 384-well plates. At $24 \mathrm{~h}$, cells were treated with various drug doses in quadruplicate wells for $72 \mathrm{~h}$. Drug treatments consisted of $1: 2$ or $1: 3$ serial dilutions. After $72 \mathrm{~h}$ of treatment, Cell Titer-Glo ${ }^{\bullet}$ (Promega) was added to the cells, and luminescence was read using the PerkinElmer EnVision Multilabel Plate Reader. Samples were normalized to untreated controls and dose response curves and IC50 values were determined using GraphPad Prism. Drug synergy was determined using CompuSyn software (ComboSyn, Inc) for Chou-Talalay analysis [17].

\section{Western blots}

Cells were plated at 2000,000 cells/well in 6 well plates and allowed to grow for 6 or $24 \mathrm{~h}$ after treatment. Treated cells were washed in PBS and lysed using RIPA lysis buffer (Thermo Fisher) supplemented with 1x Halt Protease \& Phosphatase inhibitor cocktail (Thermo Fisher). Gel electrophoresis was performed on 15 or 10well $1.5 \mathrm{~mm}$ NuPAGE 4-12\% Bis-Tris gels (Thermo Fisher). Samples were then transferred to Invitrolon PVDF transfer membranes (Invitrogen) and blocked overnight at $4{ }^{\circ} \mathrm{C}$ or $1 \mathrm{~h}$ at room temperature in blocking buffer consisting of $1 \mathrm{x}$ TBS (Quality Biological) with 0.1\% Tween ${ }^{\odot} 20$ (Thermo Fisher) and 5\% dehydrated milk. Primary antibodies were used at concentrations of 1:1000 except the GAPDH control, which was 1:5000. The secondary antibody concentration used was at a 1 : 20,000 dilution. Blots were imaged after treatment with SuperSignal West Pico Chemiluminescent substrate (Thermo Fisher) using the ChemiDoc ${ }^{\mathrm{TM}}$ Imaging System (Bio-Rad). Bands were quantified using the Image Lab ${ }^{\text {тм }}$ software (Bio-Rad). Figure 1b samples were prepared as described above and imaged using near infrared western blot detection. Blots were prepared as described above and probed with near infrared secondary antibodies IRDye $^{\oplus}$ 800CW GAR and IRDye ${ }^{\curvearrowleft}$ 680RD GAM (LICOR). Blots were imaged using LI-COR Odyssey FC and quantified using Empiria Studio ${ }^{\mathrm{TM}}$ Software (LI-COR).

\section{qRT-PCR}

Cells were plated at a concentration of 2000,000 cells/well in 6 well plates and treated for $6 \mathrm{~h}$. Total RNA was isolated from treated cells using the Qiagen RNeasy ${ }^{\circ}$ Plus Mini Kit as per manufacturer instructions. RNA samples $(1 \mu \mathrm{g})$ were reverse transcribed using the iScript Select cDNA synthesis kit
(Bio-Rad). qRT-PCR reactions were prepared in triplicate using iQ SYBR GreenSupermix (Bio-Rad) and were performed using a CFX96 Real-Time qPCR Detection System (Bio-Rad). Analysis of fold change gene expression was performed on Excel (Microsoft) spreadsheets using the Comparative $C_{\mathrm{T}}$ method $[15,18]$.

\section{Drug library screening}

KG-1a cells were plated into three 384-well plates at a concentration of 2000 cells per well using MultiFlo FX dispenser (BioTek). At $24 \mathrm{~h}$, cells were treated with 53 compounds using five doses ranging from $1 \mathrm{nM}$ to $10 \mu \mathrm{M}$ in 10 -fold increments. Compounds were added to plates from a drug screening master plate dispensed with the Acoustic Transfer System (ATS) (Biosero). Immediately following drug screen compound addition, DMSO control or GQC-05 was added to give an assay concentration of $0 \mathrm{nM}$ (DMSO), $100 \mathrm{nM}$ or $300 \mathrm{nM}$ using the ATS. After $72 \mathrm{~h}$ of treatment, Cell Titer-Glo ${ }^{\bullet}$ (Promega) was added to the cells and luminescence was read using the EnVision Multilabel Plate Reader (PerkinElmer). Samples were normalized to DMSO controls. Dose response curves and Area Under the Curve (AUC) values were determined using GraphPad Prism. Combination effects were analyzed using AUC of dose response curves and compared to DMSO controls.

\section{Combination drug treatment}

Cells were plated in 384-well plates at a concentration of 2000 cells per well using a 12-channel automated pipette (Rainin). At $24 \mathrm{~h}$, drug is added in quadruplicated wells. AML cells were treated with a matrix of concentrations of GQC-05 ranging from 20 to $600 \mathrm{nM}$ and Navitoclax ranging from $2 \mathrm{nM}-2 \mu \mathrm{M}$ for $72 \mathrm{~h}$. After $72 \mathrm{~h}$ of treatment, cell viability was assessed using Cell Titer-Glo ${ }^{\circ}$ (Promega) and luminescence was read using the EnVision Multilabel Plate Reader (PerkinElmer). Samples were normalized to no treatment controls. Dose response curves and IC50 values were determined using GraphPad Prism.

\section{Luminescence-based caspase 3/7 activity analysis}

Cells were plated in 6-well plates as described above. Aliquots $(4 \times 25 \mu \mathrm{l})$ were taken for apoptosis assays and transferred in quadruplicate to 384 well plates. CaspaseGlo $3 / 7$ (Promega) was used for the apoptosis assay to determine the activity of caspases 3 and 7, two enzymes used by cells during apoptosis. Caspase-Glo ${ }^{\circ} 3 / 7$ contains a substrate that, when cleaved by caspase 3 or 7 , produces ATP. A thermostable luciferase ATPase also present in the solution emits luminescence proportional to the amount of ATP in the sample. Luminescence was measured using the EnVision Multilabel Plate Reader (PerkinElmer). 




Fig. 1 GQC-05 induces apoptosis and DNA damage in KG1a cells but not CMK or TF-1 cells. The AML cell lines KG-1a, CMK and TF-1 were treated with vehicle (DMSO), 100 nM, 300 nM, 600 nM, or 1000 nM GQC-05 for $24 \mathrm{~h}$. a Treated cells were assayed for Caspase 3/7 activity, normalized to DMSO treated cells and shown as mean fold change $+/-$ S.D. ${ }^{*} P<0.002$, ${ }^{* *} P<0.0002$ for quadruplicate samples of three biological replicates. $\mathbf{b}$ Apoptosis analysis using flow cytometry for Annexin $V$ binding and nuclear (Vybrant Ruby) staining on AML cells treated with vehicle (DMSO) or 1000 nM GQC-05

\section{Flow cytometry}

Cells were plated and treated in 6 well plates as described above. After $24 \mathrm{~h}$ of treatment, 500,000 cell aliquots of each sample were taken, including 4 aliquots of the $10 \mu \mathrm{M}$ Etoposide positive control and 1 aliquot of each other treatment. Cells were washed twice with cell staining buffer (1\% FBS in PBS), and then resuspended in $95 \mu \mathrm{L} 1 \mathrm{X}$ Annexin V binding buffer (ThermoFisher) containing Vybrant ${ }^{\circ}$ Cell Cycle Ruby dye (ThermoFisher) and Brilliant Violet-421 ${ }^{\mathrm{TM}}$-Annexin V (BioLegend) as per manufacture's instruction. For combination studies, cells were stained with $1 \mathrm{X}$ Annexin V binding buffer (Invitrogen) with $5 \mu$ M Sytox ${ }^{\circ}$ Red dead cell stain (Life Technologies) and Annexin V Alexa Flour ${ }^{\text {rm }} 488$ conjugate (1: 20 dilution) (ThermoFisher). Instrument controls included Sytox ${ }^{\oplus}$ only, Annexin V only, or unstained sample. Samples were then incubated in the dark at room temperature for 15 min and diluted 1:10 in PBS prior to analysis. Flow cytometric analysis was performed at the University of Arizona Translational Flow Cytometry Laboratory on a FACSCanto flow cytometry system (BD Biosciences) or Guava easyCyte ${ }^{\mathrm{Th}}$ flow cytometer (Millipore). Acquired data was analyzed using FlowJo analysis software.

\section{RealTime-Glo ${ }^{\mathrm{TM}}$ Annexin V apoptosis necrosis assay (Promega)}

Cells were added to a 384 well plate at a concentration of 2500 cells per well, as per manufacturer recommendation and incubated for $18 \mathrm{~h}$ at $37^{\circ} \mathrm{C}$ with $5 \% \mathrm{CO}_{2}$. Two hours prior to drug addition, $2 \mathrm{X}$ RTG detection reagent was added to all wells. At $24 \mathrm{~h}$, drug was added in quadruplicate wells. Cells were treated with DMSO, GQC-05, Navitoclax, or GQC-05 in combination with Navitoclax. Fluorescence and luminescence were read using the EnVision Multilabel Plate Reader (PerkinElmer) at 1-h increments up to $6 \mathrm{~h}$ and another read at $24 \mathrm{~h}$. Samples were normalized to untreated controls. Dose response curves were generated using GraphPad Prism.

\section{Results}

GQC-05 induces apoptosis in AML cells

AML cell lines KG-1a, CMK, and TF-1 were treated with GQC-05 for $24 \mathrm{~h}$ and analyzed for induction of caspase $3 / 7$ as an indication of apoptosis. Caspase 3/7 activity was induced in KG-1a cells treated with $600 \mathrm{nM}$ and $1 \mu \mathrm{M}$ GQC-05 and in CMK and TF-1 cells treated with 
$1 \mu \mathrm{M}$ GQC-05 (Fig. 2a). Increased annexin V staining in was also induced by treatment with GQC-05 with the greatest increase in KG-1a cells (Fig. 2b), suggesting that KG-1a cells are more sensitive to the effects of GQC-05.

\section{GQC-05 exposure decreased MYC mRNA in AML cells}

We evaluated the activity of GQC-05 on cell viability of a panel of AML cells and determined the relative EC50 values, noting the variability in sensitivity to GQC-05 as well as the sensitivity to cytarabine, as a positive control (Table 1). Cellular response to GQC-05 varied among lines with EC50 values ranging from $4 \mathrm{nM}$ to $400 \mathrm{nM}$. We selected lines KG-1a, CMK and TF-1 for further studies due to their high expression of MYC and varied response to GQC-05. We found that GQC-05 treatment decreased expression of MYC mRNA in the AML cell lines, as well as decrease in MYC protein at $6 \mathrm{~h}$. (Fig. 1).

\section{Identification of Navitoclax as a potential cytotoxic agent} in combination with GQC-05

We next performed a small molecule combination screen using a panel of 54 cancer drugs and inhibitors to identify potential combination partners that potentiate GQC-05 activity in KG-1a cells. (Additional file 1: Table S1). Cell viability was assessed and activity of the small molecules was determined by calculating the Area Under the Curve (AUC) for each small molecule in combination with either a high or low dose of GQC-05 and compared to vehicle treated (Fig. 3a-b, Additional file 1: Table S1). In combination with $300 \mathrm{nM}$ GQC-05, the AUC of the Bcl-2/Bcl- $\mathrm{X}_{\mathrm{L}}$ inhibitor Navitoclax was significantly lower compared to vehicle treated (Fig. 3c) indicating potentiation of GQC-05 activity.

GQC-05 acts synergistically with Navitoclax in AML cells In order to evaluate the combination of GQC-05 with Navitoclax, drug synergy studies were performed on the AML cell lines KG-1a, CMK and TF-1. Dose response curves of Navitoclax indicated a shift in the EC50 when co-treated with GQC-05 compared to vehicle for all three AML cell lines (Fig. 4a). Synergy was assessed using Chou-Talalay analysis [17] and was seen across several combinatorial doses of GQC-05 and Navitoclax (Fig. 4b).

The combination of GQC-05 and Navitoclax can rapidly induce apoptosis and potentiates cytotoxicity in AML cell lines

Given that the combination of GQC-05 and Navitoclax was synergistic, we analyzed the activity of the drug combination on apoptosis. Co-treatment of AML cells with GQC-05 and $100 \mathrm{nM}$ Navitoclax for $6 \mathrm{~h}$ showed a decrease in viability at higher GQC-05 doses (Fig. 5a) and marked increase in caspase $3 / 7$ activity (Fig. 5b) and Annexin $\mathrm{V}$ staining (Fig. $5 \mathrm{c}$ ) when compared to treatment with GQC-05 alone. Kinetic studies of Annexin V




Table 1 EC50 Values of AML Cells Treated with GQC-05 or Cytarabine

\begin{tabular}{lll}
\hline Cell Line & GQC-05 $(\mathrm{nM})$ & Cytarabine $(\mathrm{nM})$ \\
\hline Molm-13 & $4 \pm 0.9$ & $4 \pm 0.9$ \\
M-07e & $70 \pm 29$ & $24 \pm 4.4$ \\
U937 & $213 \pm 109$ & $27 \pm 2.9$ \\
HEL & $203 \pm 90$ & $34 \pm 4.3$ \\
Kasumi-1 & $44 \pm 14$ & $60 \pm 57$ \\
NB4 & $113 \pm 17$ & $65 \pm 34.8$ \\
CMK & $157 \pm 26$ & $75 \pm 5$ \\
TF-1 & $128 \pm 40$ & $103 \pm 26$ \\
KG-1a & $89 \pm 30$ & $157 \pm 31$ \\
CMS & $220 \pm 48$ & $247 \pm 162$ \\
AML-193 & $321 \pm 52$ & $414 \pm 30$ \\
CMY & $104 \pm 14$ & $1588 \pm 353$ \\
THP-1 & $194 \pm 76$ & $4224 \pm 564$ \\
UT-7epo & $76 \pm 32$ & $6821 \pm 3880$ \\
\hline
\end{tabular}

binding indicated that apoptosis occurs more rapidly and at lower doses in KG-1a cells than CMK or TF-1 cells (Fig. 5d) consistent with the observation that KG1a cells are more sensitive to GQC-05. Similarly, the combination of GQC-05 and Navitoclax for $6 \mathrm{~h}$ induced an increase in PARP cleavage and an increase in $\gamma \mathrm{H} 2 \mathrm{AX}$ in KG-1a, CMK and TF-1 cells indicating potentiation of DNA damage in the AML cell lines (Fig. 5e).
Comparison of the combination of Navitoclax with GQC05 to other cytotoxic chemotherapeutic agents and inhibitors

In order to evaluate the uniqueness in response of KG-1a cells to the combination of GQC-05 and Navitoclax, we compared the activity of the combination of Navitoclax in combination with the cytotoxic agents doxorubicin and cytarabine, as well as the BET inhibitor (+)-JQ1, a bromodomain inhibitor which is also known to downregulate MYC and MYC-dependent target genes (34). Cells were co-treated with twice the IC90 doses doxorubicin, cytarabine, (+)-JQ1, or GQC-05 combined with vehicle or 100 $\mathrm{nM}$ Navitoclax for $6 \mathrm{~h}$. A decrease in viability was observed with the GQC-05 combination (Fig. 6a) as well as a more pronounced increase in caspase $3 / 7$ activity (Fig. 6b) when compared to the combination of either doxorubicin, cytarabine or (+)-JQ1 and Navitoclax.

\section{Discussion}

\section{Selective cytotoxicity of GQC-05 on a subset of AML cell} lines

One strategy to reduce expression of oncogenes is to exploit an available small molecule that binds to and stabilizes the G4 structure on the promoter leading to the downregulation of protein expression [19]. The potential for gene specificity using this approach stems from the variable structure of G-quadruplexes. Each structure is anchored by stacked guanine tetrads, but composition and length of the loops, number of tetrads, and orientation of

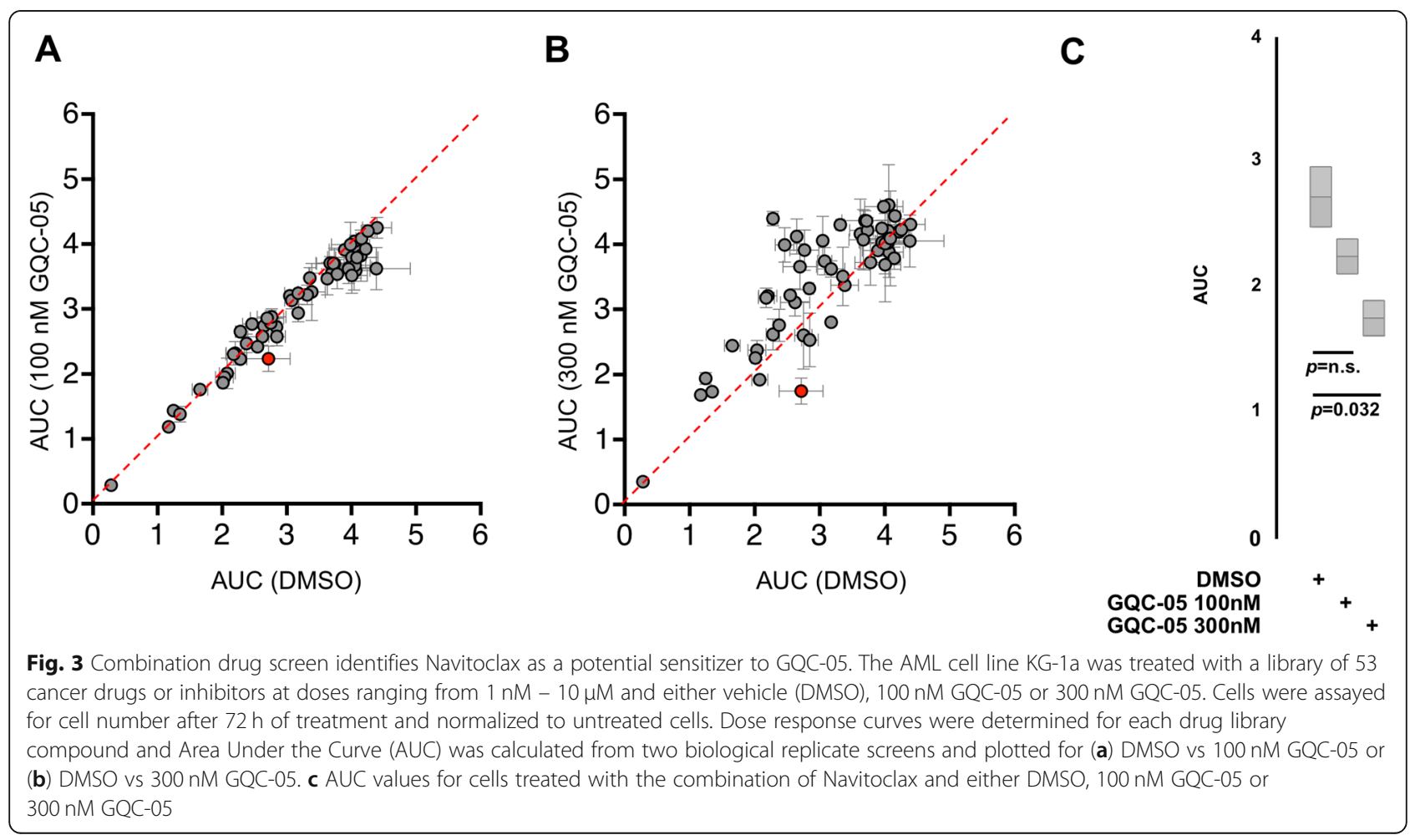






the strands can vary [20]. Small molecule inhibitors can be designed to preferentially bind specific structures. Given that G-quadruplexes form as a result of superhelical stress on DNA brought on by transcriptional unwinding [21] and oncogenes are transcribed at a higher rate in cancer cells, we hypothesized that there would be a higher presence of the G-quadruplex targets in cancer cells than in normal cells. This could reduce the effect of Gquadruplex targeted therapies on normal cells and potentially reduce toxicity in a clinical setting.

AML is the product of genetic alterations that cause dysregulation in the maturation of white blood cells, cell proliferation, cell survival, and apoptosis. Some of the most common mutations that result in AML are products of the following gene fusions: FLT3-ITD [22], AML1-ETO, PML/ $R A R a$, and PLZF/RARa [23]. Each underlying mutation of AML causes differential expression of many proto- oncogenes that could be used as potential targets (citation). Many AML mutations are correlated with increased expression of the proto-oncogene $M Y C$ making it an attractive therapeutic target in AML [12, 13, 24]. Furthermore, depletion of MYC has been shown to decrease AML cell proliferation and prolong survival of mice transplanted with AML [24]. Elevated MYC expression in AML patients is correlated with poorer prognosis.

The small molecule GQC-05 has previously been described as having G4 binding properties with a preference for the (NHE)III G-quadruplex on the $M Y C$ promoter sequence and demonstrated decreased $M Y C$ gene expression in non-Hodgkin's lymphoma cells [11]. Given that GQC-05 induced MYC decreased expression and apoptosis in lymphoma cells, we investigated whether it would have similar antiproliferative activity on AML cells. 
A
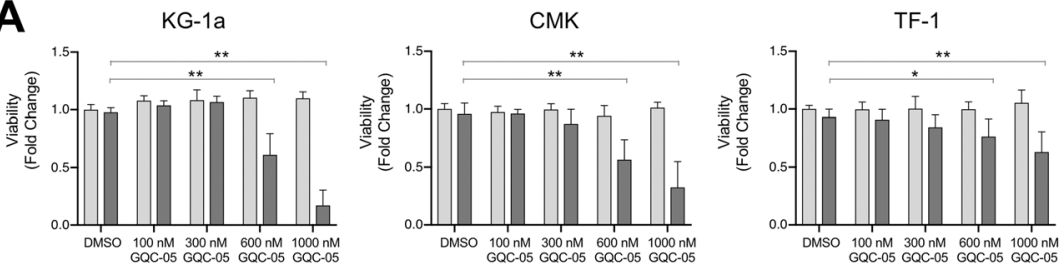

B


$\square$ GQC-05

- GQC-05+

GQC-05+
$100 \mathrm{nM}$ Navitoclax
C

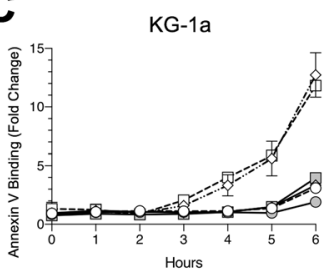

CMK
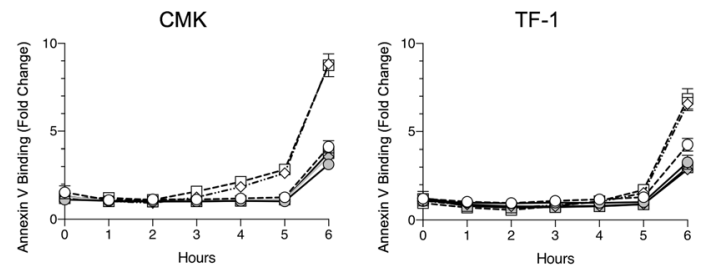

-o. DMSO + $100 \mathrm{nM}$ Navitoclax

$\approx 600 \mathrm{nM} \mathrm{GQC-05} \quad-600 \mathrm{nM}$ GQC-05 + $100 \mathrm{nM}$ Navitoclax

D


E

KG-1a

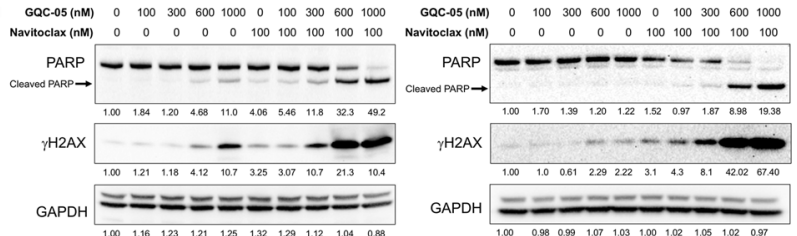

TF-1

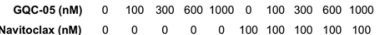

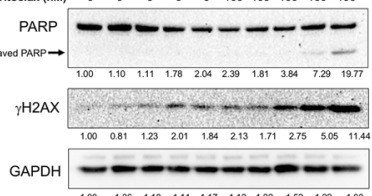

Fig. 5 (See legend on next page.) 
(See figure on previous page.)

Fig. 5 The combination of GQC-05 and Navitoclax induces rapid apoptosis, DNA damage, and potentiates cytotoxicity in AML cell lines. The AML cell lines KG-1a, CMK and TF-1 were treated with vehicle (DMSO) 100, 300, 600 and 1000 nM GQC-05 ranging in combination with either vehicle (DMSO) or $100 \mathrm{nM}$ Navitoclax for $6 \mathrm{~h}$. a-b Treated cells were assayed for (a) cell number and (b) Caspase 3/7 activity after $6 \mathrm{~h}$ of each treatment, normalized to vehicle treated cells and plotted as plotted as mean $+/-\mathrm{SD} .{ }^{*} P<0.005,{ }^{* *} P<0.0001$ for quadruplicate samples of three biological replicates. c Kinetic analysis of Annexin V binding upon treatment of AML cells with GQC-05 and either vehicle (DMSO) or $100 \mathrm{nM}$ Navitoclax. Graphs are representative of two biological replicates using triplicate wells, normalized to Time 0 and plotted as mean $+/-$ SD. $\mathbf{d}$ Dual color flow cytometric analysis using Alexafluor 488-Annexin $V$ and SytoxRed staining of treated cells. e Western immunoblotting of whole cell lysates from KG-1a, CMK, and TF-1 cells analyzed for expression of cleaved PARP and $\gamma \mathrm{H} 2 \mathrm{AX}$. GADPH is shown as a loading control. Band intensities for cleaved PARP and $\mathrm{YH} 2 \mathrm{AX}$ were calculated and normalized to GAPDH and vehicle controls

Treatment of 14 AML cell lines with GQC-05 showed a wide range of cytotoxic activity (Table 1 ) and $M Y C$ expression. Three cell lines KG-1a, CMK and TF-1 were selected for further analysis based on their varied sensitivity to GQC-05 and expression of MYC. The promyelobalstic cell line KG-1a and the erythroblast cell line TF-1 were both derived from adult AML patients $[25,26]$. The CMK cell line was derived from a megakaryoblastic pediatric leukemia patient with Down syndrome [15]. The KG-1a cell line was the most sensitive of these three lines to the cytotoxic effects of GQC-05 (Table 1). Treatment with GQC-05 showed more substantial knockdown of $M Y C$ mRNA and MYC protein in the KG-1a cell lines after 6-h treatment compared to CMK and TF-1 cells (Fig. 2).
GQC-05 also induced apoptosis after $24 \mathrm{~h}$ of exposure in KG-1a cells, which was not as notable in CMK and TF-1 cells (Fig. 1a). Moreover, GQC-05 induced H2AX phosphorylation in KG-1a cells indicating that DNA damage could also be induced by GQC- 05 treatment. These data suggest that reduction of MYC expression and the cytotoxic effects of GQC-05 can vary across AML cell lines and that MYC expression may not necessarily correlate to GQC-05 activity as there are likely promoters with which GQC-05 interacts in addition to MYC. Furthermore, activity of GQC-05 involves both apoptosis and DNA damage in this cellular context.

Recently, a G-quadruplex stabilizer APTO-253 was described that down regulated MYC expression and also

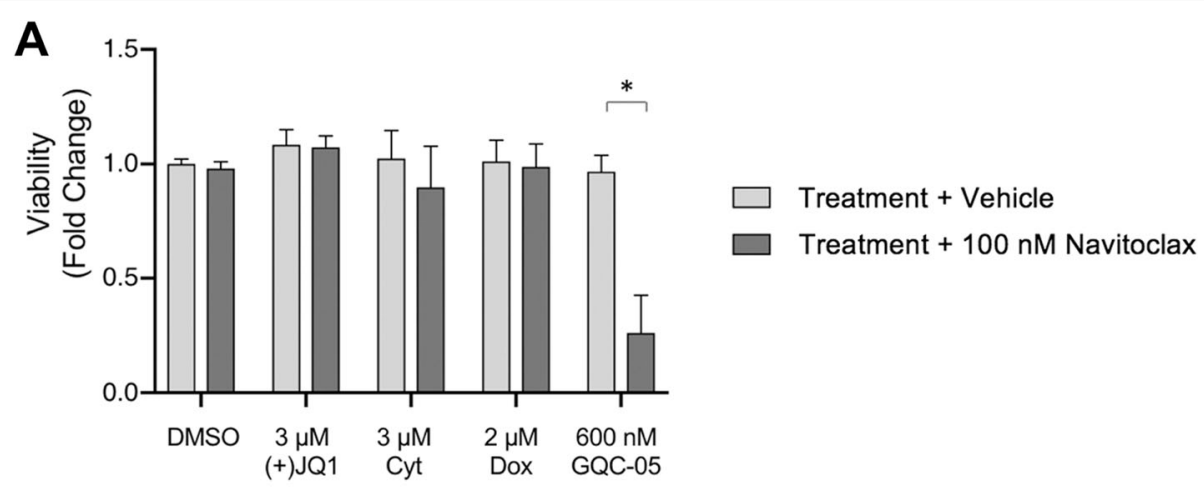

B



Treatment + Vehicle

Treatment + $100 \mathrm{nM}$ Navitoclax

Fig. 6 Navitoclax potentiates apoptosis and cytotoxicity greater in combination with GQC-05 than with doxorubicin, cytarabine, or (+)-JQ1. The AML cell lines KG-1a was treated with vehicle (DMSO), $3 \mu \mathrm{M}$ (+)-JQ1, $3 \mu \mathrm{M}$ Cytarabine (Cyt), $2 \mu \mathrm{M}$ Doxorubicin (Dox) or 600 nM GQC-05 in combination with either vehicle (DMSO) or $100 \mathrm{nM}$ Navitoclax for $6 \mathrm{~h}$. a-b Treated cells were assayed for (a) cell number and (b) Caspase 3/7 activity after $6 \mathrm{~h}$ and normalized to vehicle treated cells. Quadruplicate wells were measured for each experiment and the graph of three biological replicate was plotted as mean $+/-\mathrm{SD}$. ${ }^{*} P<0.0001$ 
induced DNA damage in AML cells [27], similar to what was observed by GQC-05. APTO-253, which is presently in clinical studies, was also described as inducing p21 and promoting cell cycle arrest. Although there are similarities in the activity of these two G-quadruplex binding small molecules, structurally APTO-253 is a 2-indolyl imidazole (4,5-d) phenanthroline derivative, which differs from the ellipticine GQC-05.

We sought to identify small molecules that could potentiate the activity of GQC-05, and performed combination drug screening using a cohort of inhibitors and identified the Bcl-2/Bcl- $\mathrm{X}_{\mathrm{L}}$ inhibitor Navitoclax as a potentiator of GQC-05 (Fig. 3). Further analysis showed that combinations of Navitoclax and GQC-05 were synergistic (Fig. 4) and rapidly induced apoptosis and DNA damage (Fig. 5). We next aimed to determine if other agents that caused either DNA damage or decreased MYC expression could induce the rapid apoptosis and decreased cell viability when combined with Navitoclax similar to that with GQC-05. Both doxorubicin [28] and cytarabine [29] have been previously shown to induce damage DNA [28, 29], while the BET inhibitor (+)-JQ1 can down-regulate $M Y C$ expression [30]. Interestingly, the rapid apoptosis was predominantly seen with the GQC-05/Navitoclax combination and mildly with the cytarabine/Navitoclax combination compared to Navitoclax combinations with either doxorubicin or the BET inhibitor (+)-JQ1. The rapid decrease in cell viability was seen with only the GQC-05/Navitoclax combination, thus indicating that GQC-05 may possess additional mechanisms of cytotoxic activity. GQC-05 has previously shown to have binding activity to other G-quadruplex sites such as Bcl-2, HIF- $1 \alpha$, hTERT, PDGF-A, PDGF-R $\beta$, and potentially others [11]. The lack of cytotoxicity and apoptosis in the (+)-JQ1/Navitoclax combination further demonstrates that the synergistic effects of GQC-05/ Navitoclax combination may not be MYC mediated.

Navitoclax is a potent inhibitor of both Bcl-2 and Bcl$\mathrm{X}_{\mathrm{L}}$ activity [31] and has previously been shown to be an effective partner for cytotoxic agents including cytarabine in T-ALL cells [32] and solid tumor cell lines [33]. Our data suggests that the activity of GQC-05 in combination with $\mathrm{Bcl}-2 / \mathrm{Bcl}-\mathrm{X}_{\mathrm{L}}$ inhibition promotes a more pronounced and rapid cytotoxic event in AML cells. This result is supported by a recent study using a combination of GQC-05 and a C-quadruplex (i-motif) binding small molecule that down-regulated $\mathrm{Bcl}-2$ showed increased activity over GQC-05 alone in diffuse large Bcell lymphoma [34]. Another recent study showed that GQC-05 can bind to the G-quadruplex structures on the pre-mRNA of Bcl-X thus affecting RNA splicing resulting in antagonism of the anti-apoptotic Bcl- $\mathrm{X}_{\mathrm{L}}$ and activation of the pro-apoptotic form Bcl- $\mathrm{X}_{\mathrm{S}}$ [35]. This finding would suggest that GQC-05 could reduce the expression $\mathrm{Bcl}-\mathrm{X}_{\mathrm{L}}$ thereby lowering the threshold for Bcl- $\mathrm{X}_{\mathrm{L}}$ inhibition by Navitoclax, and lead to the observed increase activity of the combined agents.

GQC-05 illustrated a marked increase in DNA damage and apoptosis in AML cell lines. Though, direct stabilization of the MYC NHEIII G-quadruplex by GQC-05 would lead to decreased MYC expression it's also likely that GQC-05 may bind other G-quadruplex structures or DNA motifs, potentially leading to additional DNA damage.

\section{Conclusions}

In summary, our results suggest that the G-quadruplex stabilizing compound GQC-05 has varied activity in the panel of AML cells tested. GQC-05 induced DNA damage and apoptosis, both of which are enhanced by with the addition of the $\mathrm{Bcl}-2 / \mathrm{Bcl}-\mathrm{X}_{\mathrm{L}}$ inhibitor Navitoclax. The combination effect with GQC-05 and Navitoclax is more rapid and pronounced than the combination effects of GQC-05 and other chemotherapeutic agents that are currently being used to treat AML patients. GQC-05 down regulates MYC at the mRNA and protein level, though the pronounced combinatory effects are most likely mediated through other binding sites. These additional properties would need to be further characterized to better understand the mechanism of GQC-05 and possibly similar compounds that can stabilize G-quadruplexes.

\section{Supplementary information}

Supplementary information accompanies this paper at https://doi.org/10. 1186/s12885-019-6464-9.

Additional file 1: Table S1. AUC values for Combination Drug Screen with GQC-05 in KG-1a Cells.

\section{Abbreviations}

AML: Acute Myeloid Leukemia; ATS: Acoustic Transfer System; AUC: Area Under the Curve; DMSO: Dimethyl Sulfoxide; G4: G-quadruplex; GMCSF: Granulocyte Macrophage Colony-Stimulating Factor; STR: Short Tandem Repeat

\section{Acknowledgments}

This manuscript is dedicated in memoriam of Robert J. Arceci. The authors wish to thank John Couvaras for his technical assistance and Dr. Mrinalani Kala of the University of Arizona Translational Flow Cytometry Laboratory for her aid with the flow cytometric analysis.

\section{Authors' contributions}

DOA, JJM, MAT RJA, CW, LHH, and VG designed the experimental approach. JJM, MAT, DWL, AP, and DOA collected data. JJM, MAT, DHW, and DOA analyzed the data. All authors were involved in interpretation of the data. JJM, MAT, CW and DOA wrote the manuscript. All authors have read and approved the final manuscript.

\section{Funding}

This work was funded by Sharon D. Lund Foundation, University of Arizona COM-P Department of Child Health Mission Support, the Phoenix Children's Hospital Foundation, the St. Baldrick's Foundation and 5RO1CA177585 (LHH). The funding bodies had no role in the design of the study and collection, analysis, interpretation of data, or in writing this manuscript. 


\section{Availability of data and materials}

All data generated or analyzed during this study are included in this published article and its supplementary information files.

Ethics approval and consent to participate Not applicable.

\section{Consent for publication}

Not applicable

\section{Competing interests}

Laurence Hurley and Vijay Gokhale have a financial interest in Reglagene, a G-quadruplex- targeting company. The other authors declare no competing interests.

\section{Author details}

${ }^{1}$ The Institute of Molecular Medicine at Phoenix Children's Hospital, $475 \mathrm{~N}$ 5th Street, Phoenix, AZ 85004, USA. ${ }^{2}$ Department of Child Health, The University of Arizona College of Medicine-Phoenix, 475 N 5th Street, Phoenix, AZ 85004, USA. ${ }^{3}$ School of Life Sciences, Arizona State University, Tempe, AZ, USA. ${ }^{4}$ Present Address: Department of Molecular \& Medical Genetics, Oregon Health and Science University, Portland, OR 97239, USA. ${ }^{5}$ Center for Cancer and Blood Disorders, Phoenix Children's Hospital, Phoenix, AZ, USA. ${ }^{6}$ University of Arizona College of Pharmacy, Tucson, AZ, USA. ${ }^{7}$ Present Address: Systems Oncology, Scottsdale, AZ 85255, USA.

Received: 2 August 2019 Accepted: 15 December 2019 Published online: 27 December 2019

\section{References}

1. Dohner H, Weisdorf DJ, Bloomfield CD. Acute Myeloid Leukemia. N Engl J Med. 2015;373(12):1136-52

2. Li S, Mason CE, Melnick A. Genetic and epigenetic heterogeneity in acute myeloid leukemia. Curr Opin Genet Dev. 2016;36:100-6.

3. Bolouri H, Farrar JE, Triche T Jr, Ries RE, Lim EL, Alonzo TA, Ma Y, Moore R, Mungall AJ, Marra MA, et al. The molecular landscape of pediatric acute myeloid leukemia reveals recurrent structural alterations and age-specific mutational interactions. Nat Med. 2018;24(1):103-12.

4. Kavianpour M, Ahmadzadeh A, Shahrabi S, Saki N. Significance of oncogenes and tumor suppressor genes in AML prognosis. Tumour Biol. 2016;37(8):10041-52

5. Farrar JE, Schuback HL, Ries RE, Wai D, Hampton OA, Trevino LR, Alonzo TA, Guidry Auvil JM, Davidsen TM, Gesuwan P, et al. Genomic profiling of pediatric acute myeloid leukemia reveals a changing mutational landscape from disease diagnosis to relapse. Cancer Res. 2016;76(8):2197-205.

6. DiNardo CD, Cortes JE. Mutations in AML: prognostic and therapeutic implications. Hematol Am Soc Hematol Educ Program. 2016;2016(1):348-55.

7. Eddy J, Maizels N. Gene function correlates with potential for G4 DNA formation in the human genome. Nucleic Acids Res. 2006;34(14):3887-96.

8. Rhodes D, Lipps HJ. G-quadruplexes and their regulatory roles in biology. Nucleic Acids Res. 2015;43(18):8627-37.

9. Bochman ML, Paeschke K, Zakian VA. DNA secondary structures: stability and function of G-quadruplex structures. Nat Rev Genet. 2012;13(11):770-80

10. Siddiqui-Jain A, Grand CL, Bearss DJ, Hurley LH. Direct evidence for a Gquadruplex in a promoter region and its targeting with a small molecule to repress c-MYC transcription. Proc Natl Acad Sci U S A. 2002;99(18):11593-8.

11. Brown RV, Danford FL, Gokhale V, Hurley LH, Brooks TA. Demonstration that drugtargeted down-regulation of MYC in non-Hodgkins lymphoma is directly mediated through the promoter G-quadruplex. J Biol Chem. 2011;286(47):41018-27.

12. Vita $M$, Henriksson M. The Myc oncoprotein as a therapeutic target for human cancer. Semin Cancer Biol. 2006;16(4):318-30.

13. Prochownik EV, Vogt PK Therapeutic targeting of Myc. Genes Cancer. 2010;1 (6):650-9.

14. Miura N, Sato T, Fuse A, Okimoto Y, Kinugawa N, Horie H, Ota S, Kakuda H, Yokoe $H$, Miya T, et al. Establishment of a new human megakaryoblastic cell line, CMY, with chromosome 17p abnormalities. Int J Mol Med. 1998;1(3):559-63.

15. Komatsu N, Suda T, Moroi M, Tokuyama N, Sakata Y, Okada M, Nishida T, Hirai Y, Sato T, Fuse A, et al. Growth and differentiation of a human megakaryoblastic cell line, CMK. Blood. 1989;74(1):42-8.

16. Sato T, Sekine $H$, Kakuda H, Miura N, Sunohara M, Fuse A. HIV infection of megakaryocytic cell lines. Leuk Lymphoma. 2000;36(3-4):397-404.
17. Chou TC, Talalay P. Quantitative analysis of dose-effect relationships: the combined effects of multiple drugs or enzyme inhibitors. Adv Enzym Regul. 1984;22:27-55.

18. Schmittgen TD, Livak KJ. Analyzing real-time PCR data by the comparative C(T) method. Nat Protoc. 2008;3(6):1101-8.

19. Brooks TA, Hurley LH. Targeting MYC expression through G-Quadruplexes. Genes Cancer. 2010;1(6):641-9.

20. Brooks TA, Kendrick S, Hurley L. Making sense of G-quadruplex and i-motif functions in oncogene promoters. FEBS J. 2010;277(17):3459-69.

21. Sun D, Hurley LH. The importance of negative superhelicity in inducing the formation of G-quadruplex and i-motif structures in the c-Myc promoter: implications for drug targeting and control of gene expression. J Med Chem. 2009;52(9):2863-74.

22. Gilliland DG, Griffin JD. The roles of FLT3 in hematopoiesis and leukemia. Blood. 2002;100(5):1532-42.

23. Muller-Tidow C, Steffen B, Cauvet T, Tickenbrock L, Ji P, Diederichs S, Sargin B, Kohler G, Stelljes M, Puccetti E, et al. Translocation products in acute myeloid leukemia activate the Wnt signaling pathway in hematopoietic cells. Mol Cell Biol. 2004;24(7):2890-904.

24. Brondfield S, Umesh S, Corella A, Zuber J, Rappaport AR, Gaillard C, Lowe SW, Goga A, Kogan SC. Direct and indirect targeting of MYC to treat acute myeloid leukemia. Cancer Chemother Pharmacol. 2015;76(1):35-46.

25. Koeffler HP. Induction of differentiation of human acute myelogenous leukemia cells: therapeutic implications. Blood. 1983;62(4):709-21.

26. Kitamura T, Tange T, Terasawa T, Chiba S, Kuwaki T, Miyagawa K, Piao YF, Miyazono K, Urabe A, Takaku F. Establishment and characterization of a unique human cell line that proliferates dependently on GM-CSF, IL-3, or erythropoietin. J Cell Physiol. 1989;140(2):323-34.

27. Local A, Zhang H, Benbatoul KD, Folger P, Sheng X, Tsai CY, Howell SB, Rice WG. APTO-253 stabilizes G-quadruplex DNA, inhibits MYC expression, and induces DNA damage in acute myeloid leukemia cells. Mol Cancer Ther. 2018;17(6):1177-86.

28. Cruet-Hennequart S, Prendergast AM, Shaw G, Barry FP, Carty MP. Doxorubicin induces the DNA damage response in cultured human mesenchymal stem cells. Int J Hematol. 2012;96(5):649-56.

29. Ma J, Li X, Su Y, Zhao J, Luedtke DA, Epshteyn V, Edwards H, Wang G, Wang $Z$, Chu R, et al. Mechanisms responsible for the synergistic antileukemic interactions between ATR inhibition and cytarabine in acute myeloid leukemia cells. Sci Rep. 2017;7:41950.

30. Filippakopoulos P, Qi J, Picaud S, Shen Y, Smith WB, Fedorov O, Morse EM, Keates T, Hickman TT, Felletar I, et al. Selective inhibition of BET bromodomains. Nature. 2010:468(7327):1067-73.

31. Tse C, Shoemaker AR, Adickes J, Anderson MG, Chen J, Jin S, Johnson EF, Marsh KC, Mitten MJ, Nimmer P, et al. ABT-263: a potent and orally bioavailable BCl-2 family inhibitor. Cancer Res. 2008;68(9):3421-8.

32. Anderson NM, Harrold I, Mansour MR, Sanda T, McKeown M, Nagykary N, Bradner JE, Lan Zhang G, Look AT, Feng H. BCL2-specific inhibitor ABT-199 synergizes strongly with cytarabine against the early immature LOUCY cell line but not more-differentiated T-ALL cell lines. Leukemia. 2014;28(5):1145-8.

33. Chen J, Jin S, Abraham V, Huang X, Liu B, Mitten MJ, Nimmer P, Lin X, Smith $\mathrm{M}$, Shen $\mathrm{Y}$, et al. The $\mathrm{BCl}-2 / \mathrm{BCl}-\mathrm{X}(\mathrm{L}) / \mathrm{BCl}-\mathrm{w}$ inhibitor, navitoclax, enhances the activity of chemotherapeutic agents in vitro and in vivo. Mol Cancer Ther. 2011:10(12):2340-9.

34. Kendrick S, Muranyi A, Gokhale V, Hurley LH, Rimsza LM. Simultaneous drug targeting of the promoter MYC G-Quadruplex and BCL2 i-motif in diffuse large B-cell lymphoma delays tumor growth. J Med Chem. 2017;60(15):6587-97.

35. Weldon C, Dacanay JG, Gokhale V, Boddupally PVL, Behm-Ansmant I, Burley GA, Branlant C, Hurley LH, Dominguez C, Eperon IC. Specific G-quadruplex ligands modulate the alternative splicing of BCl-X. Nucleic Acids Res. 2018; 46(2):886-96.

\section{Publisher's Note}

Springer Nature remains neutral with regard to jurisdictional claims in published maps and institutional affiliations. 